\title{
Influence des souches seules et associées de L. bulgaricus et Str. thermophilus ainsi que des traitements du lait sur la production d'aldéhyde acétique dans le yaourt
}

\author{
par \\ V. BOTTAZZI, B. BATTISTOTTI \\ et G. MONTESCANI \\ Istituto di Microbiologia Lattiero-casearia, Università' Cattolica \\ del Sacro Cuore, Piacenza (Italie)
}

Le yaourt, dans sa zone d'origine qui est la péninsule balkanique, était préparé avec du lait de chèvre et à l'aide de cultures naturelles.

Les recherches de Grigoroff [1] et de Kuntze [2], conduites au début de ce siècle, ainsi que les recherches successives de OrlaJensen [3], nous ont indiqué que les ferments lactiques de telles cultures étaient représentés par Lactobacillus jugurti et Lactobacillus bulgaricus.

Cependant Metchnikoff indiqua que le produit original était principalement obtenu avec des lactobacilles du type bulgaricus.

Depuis que le yaourt de production artisanale est devenu produit industriel, avec, en conséquence, une large diffusion dans tous les pays d'Europe, il a perdu diverses caractéristiques originelles pour devenir le résultat d'une combinaison microbienne différente et d'un processus industriel.

La culture naturelle est généralement substituée par une association de bacilles et de streptocoques thermophiles et, ces derniers temps, de nombreuses recherches ont été réservées à l'explication du rôle que précisément $L$. bulgaricus et Str. thermophilus exercent dans la production et sur la qualité du yaourt actuel.

Mais la nature chimique de la « saveur du yaourt » n'a certainement pas encore été approfondie. Pette et Lolkema [4] ont démontré que l'aldéhyde acétique était le composant principal de la saveur du yaourt et, successivement, Bottazzi et Dellaglio [5] ont indiqué que $L$. bulgaricus était le responsable de sa production. 
Tout ceci ne peut être considéré comme suffisant en ce qui concerne la diversité des "saveurs du yaourt » et, par conséquent, des types de lait utilisés et de la combinaison des cultures employées.

En fait, on peut ainsi expliquer comment Schulz et Hingst [6] puis Schulz, Voss et Kley [7], dans le yaourt de lait de chèvre même de bonne qualité, n'ont pas rencontré une quantité appréciable d'aldéhydes acétiques et comment Turcic, Rasic et Canic [8] donnent de l'importance même aux acides gras volatils.

Reste donc établi le fait que dans le yaourt de production industrielle obtenu avec $L$. bulgaricus et Str. thermophilus, l'aldéhyde acétique est l'unique substance volatile (Görner, Palo et Bertan [9]) produite en quantité significative pendant sa préparation.

Notre étude, conduite suivant la méthode "Head Space » et examens " chromatographies en phase gazeuse " de l'aldéhyde acétique, a pour but de fournir une contribution à la connaissance de l'influence des souches seules et associées composant la culture pour yaourt et aussi sur les traitements thermiques du lait dans la formation de la «saveur du yaourt » ayant pour base l'aldéhyde acétique.

\section{METHODES}

a) Substrat pour l'isolement des souches :

- pour les formes bacillaires le milieu (MRS) [10] avec incubation à $42^{\circ} \mathrm{C}$ pendant $48 \mathrm{~h}$ dans une ambiance d'azote (pour 90 p. 100) et de $\mathrm{CO}_{2}$,

- pour les formes streptocoques : le milieu agar-sérum de Politi [11] avec incubation à $42^{\circ} \mathrm{C}$ pendant $72 \mathrm{~h}$.

b) Fermentation des sucres : l'essai fut effectué avec le substrat suivant : peptone $10 \mathrm{~g}$, extrait de levure $5 \mathrm{~g}, \mathrm{MgSO}_{*} 0,05 \mathrm{~g}$, solution de bromo-cresol pourpre $2,8 \mathrm{ml}$ (d'une solution alcoolisée à 1,6 p. 100), eau $1000 \mathrm{ml}$, pH final 6,8 .

Les sucres furent ajoutés à raison de 1 p. 100 .

c) Composition de DNA : les différentes souches après développement sur bouillon MRS, furent traitées pour l'extraction du DNA, suivant la technique de Marmur [12]. De l'allure de la courbe de dénaturation thermique du DNA et de l'application de la formule de De Ley [13] on estime le pourcentage de guanine et de cétosine présentes.

d) Test sérologique : on a suivi la technique indiquée par Sharpe [14] employant le lapin.

e) Evaluation de l'aldéhyde acétique : l'évaluation de ce composé monocarbonilique fut faite directement par chromatographie selon la technique "Head Space " basée sur l'indication de Jennings, Viljhalmsson et Dunkley [15] et de Bassette, Ozeris et Whitnah [16]. 
On a fait développer les cultures dans $400 \mathrm{ml}$ de lait UHT ou lait autoclavé ou encore de lait UHT traité à $90^{\circ} \mathrm{C}$ pendant $30 \mathrm{mn}$, partagé en bouteilles de $500 \mathrm{ml}$, fermées après inoculation, avec des bouchons de caoutchouc et incubées à la température de $42^{\circ} \mathrm{C}$.

La période d'incubation terminée, on a procédé à la cassure du caillot par agitation et on a mis les bouteilles au bain-marie à $55^{\circ} \mathrm{C}$ pendant $30 \mathrm{mn}$.

Après réchauffement, on procède à une nouvelle agitation des échantillons et avec une seringue spéciale Hamilton on prélève $2 \mathrm{ml}$ des gaz " Head Space » qui sont immédiatement injectés dans l'appareil ; la seringue, à chaque prélèvement de gaz, fut néanmoins maintenue à $55^{\circ} \mathrm{C}$ par circulation d'eau chaude afin de réduire la condensation des composants volatils dans la seringue.

Pour la séparation des composants volatils on a employé une colonne 1,2,3, TRIS ( 2 cianoetossipropane) sur Chromosorb W (80-100 mesh) ayant $2 \mathrm{~m}$ de long et $4 \mathrm{~mm}$ de diamètre, température de la colonne $80^{\circ} \mathrm{C}$, injecteur $180^{\circ} \mathrm{C}$, gaz de transport $\mathrm{N}_{2}(25 \mathrm{ml} / \mathrm{mn})$.

Les cultures ont enfin été jugées sur la base des mesures effectuées au planimètre des surfaces des pics des résultantes chromatographiques.

\section{RESULTATS ET DISCUSSION}

Des divers échantillons de yaourt naturel communément trouvés dans le commerce, on a isolé diverses souches de bacilles et de streptocoques et, pour le développement de travail en question, après une première série d'essais préliminaires, on en a choisi 10 de celles-ci, dont précisément 7 de forme bacillaire et 3 streptocoques.

Les caractéristiques générales de ces souches sont réunies dans le tableau 1 dont il apparaît clairement que toutes celles de morphologie bacillaire appartiennent pour leur capacité à fermenter les sucres, par sérologie et pour leur contenu de guanine et cétosine du DNA p. $100(\mathrm{G}+\mathrm{C})$ à L. bulgaricus et les streptocoques à Str. thermophilus ; les souches de L. bulgaricus, prises en considération, sont suffisamment constantes dans leur capacité à fermenter les sucres et dans le p. $100(C+G)$ présentent des variations parfaitement normales, pour cela, on peut dire qu'elles représentent un groupe homogène et répondant certainement au but de notre recherche.

\section{A) Caractéristiques de la souche et influence de milieu sur la capacité à produire l'aldéhyde acétique}

La capacité de la part des souches isolées de produire de l'aldéhyde acétique a été essayée aussi dans le lait re-stérilisé en autoclave. 
TABLEAU 1. - Caractéristiques générales des souches

\begin{tabular}{|c|c|c|c|c|c|c|c|c|c|c|c|c|c|c|c|}
\hline \multirow[b]{2}{*}{ Souches } & \multirow{2}{*}{$\begin{array}{l}\frac{0}{00} \\
0 \\
0 \\
\frac{1}{2} \\
\frac{0}{2} \\
\dot{z}\end{array}$} & \multicolumn{10}{|c|}{ Fermentation de } & \multirow{2}{*}{ 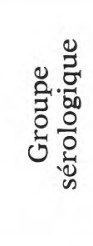 } & \multirow[b]{2}{*}{$\operatorname{Tm}$} & \multirow[b]{2}{*}{$\begin{array}{c}\text { p. } 100 \\
(G+C)\end{array}$} & \multirow[b]{2}{*}{ Classification } \\
\hline & & 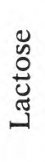 & 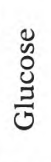 & 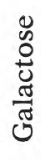 & 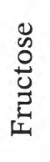 & 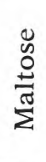 & $\frac{\mathscr{J}}{\stackrel{\Xi}{0}}$ & 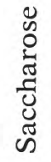 & 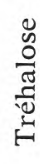 & $\begin{array}{l}0 \\
0 \\
0 \\
.0 \\
0 \\
\frac{\pi}{4}\end{array}$ & $\begin{array}{l}0 \\
0 \\
0 \\
0 \\
0 \\
0 \\
0\end{array}$ & & & & \\
\hline $\begin{array}{ll}\text { CHL } & 12 \\
\text { CHL } & 21 \\
\text { SH } & 37 \\
\text { PRA } & 10 \\
\text { PRA } & 55 \\
\text { MOR } & 72 \\
\text { MOR } & 77 \\
\text { STR } & 45 \\
\text { STR } & 63 \\
\text { ZOL } & 29\end{array}$ & $\begin{array}{l}\text { B (*) } \\
\text { B } \\
\text { B } \\
\text { B } \\
\text { B } \\
\text { B } \\
\text { B } \\
\text { S } \\
\text { S } \\
\text { S }\end{array}$ & $\begin{array}{l}+ \\
+ \\
+ \\
+ \\
+ \\
+ \\
+ \\
+ \\
+ \\
+\end{array}$ & $\begin{array}{l}+ \\
+ \\
+ \\
+ \\
+ \\
+ \\
+ \\
+ \\
+ \\
+\end{array}$ & $\begin{array}{l}- \\
- \\
+ \\
- \\
- \\
+ \\
+ \\
+\end{array}$ & $\begin{array}{l}- \\
+ \\
+ \\
+ \\
+ \\
+ \\
+ \\
+ \\
+ \\
+\end{array}$ & $\begin{array}{l}- \\
- \\
- \\
- \\
- \\
- \\
-\end{array}$ & $\begin{array}{l}- \\
- \\
- \\
- \\
-\end{array}$ & $\begin{array}{l}- \\
- \\
- \\
- \\
- \\
+ \\
+ \\
+\end{array}$ & $\begin{array}{l}- \\
- \\
- \\
- \\
- \\
- \\
-\end{array}$ & $\begin{array}{l}- \\
- \\
- \\
- \\
-\end{array}$ & $\begin{array}{l}- \\
- \\
- \\
- \\
-\end{array}$ & $\begin{array}{l}E \\
E \\
E \\
E \\
E \\
E \\
E \\
\operatorname{sr}\left({ }^{* *}\right) \\
\text { sr } \\
\text { sr }\end{array}$ & $\begin{array}{l}89,15 \\
90,35 \\
90,30 \\
91,00 \\
90,00 \\
90,45 \\
90,40\end{array}$ & $\begin{array}{l}48,20 \\
51,10 \\
51,00 \\
52,70 \\
50,30 \\
51,40 \\
51,30\end{array}$ & 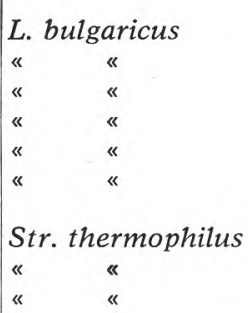 \\
\hline
\end{tabular}

(*) $\mathrm{B}=$ morphologie bacillaire

$\mathrm{S}=$ morphologie à streptocoques.

$\left.{ }^{(* *}\right) \mathrm{sr}=$ sans réaction 
Dans le lait UHT les souches de L. bulgaricus PRA 10, PRA 55, MOR 72 et MOR 77 se sont révélées faiblement productrices d'aldéhyde acétique tandis que les souches CHL 12, SH 37 et CHL 21 ont démontré leur pouvoir d'en accumuler des quantités vraiment élevées.

Il faut noter que, semblables aux premières, celles que nous avons isolées, des divers échantillons de yaourt, sont pour la plupart produites par des maisons commerciales différentes prises en considération.

De même, les souches de Str. thermophilus dans le lait UHT se sont révélées faiblement productrices d'aldéhyde acétique, un exemple de ces différences est clairement visible dans la figure 1 où on a reporté les chromatogrammes de quelques souches.

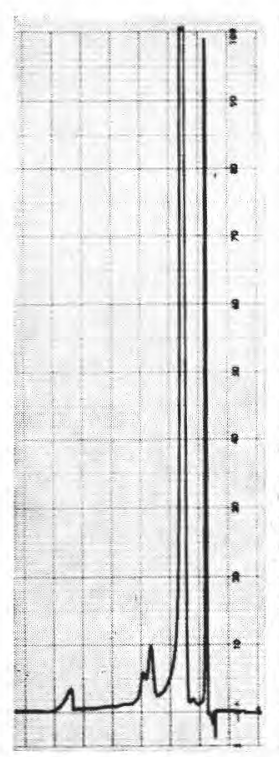

4

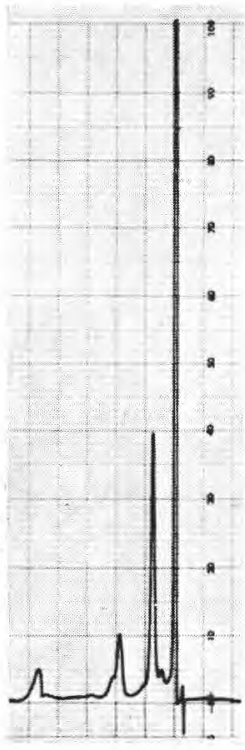

3
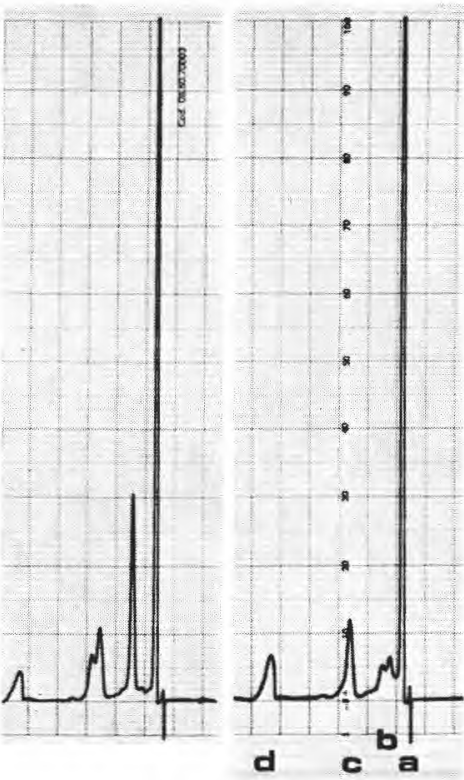

2

fig. 1

Production d'aldéhyde acétique en lait UHT par chaque souche

1 = test-lait UHT;

$2=$ PRA 55 (L. bulgaricus);

$3=$ STR 45 (Str. thermophilus);

$4=$ CHL 12 (L. bulgaricus);

$\mathrm{a}=$ pas identifié ;

$\mathrm{b}=$ aldéhyde acétique

$\mathrm{c}=$ éthanol et acétone ;

$\mathrm{d}=$ pas identifié.

(sensibilité $2 \mathrm{x}$ ). 
La comparaison entre les souches CHL 12 et PRA 55 est certainement significative, pour toutes les deux de l'espèce $L$. bulgaricus la production d'aldéhyde acétique, bien que vraiment différente, est obtenue avec le même substrat et dans les mêmes conditions de développement.

Quand on passe à une opération avec le lait UHT re-stérilisé en autoclave pendant $20 \mathrm{mn}$ à $111^{\circ} \mathrm{C}$ on note pour quelques-unes de ces souches un comportement sensiblement différent.

On a constaté en effet qu'il y a des souches comme PRA 10 et PRA 55 qui, dans le lait UHT autoclavé sont capables, à la différence de ce qui a lieu dans le lait UHT, d'accumuler de grandes quantités d'aldéhyde acétique tandis que les autres, comme CHL 12, qui étaient déjà fortement productrices dans le lait UHT ne montrent pas de différences significatives.

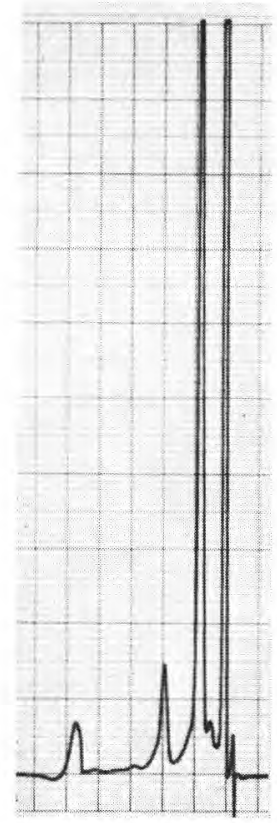

3

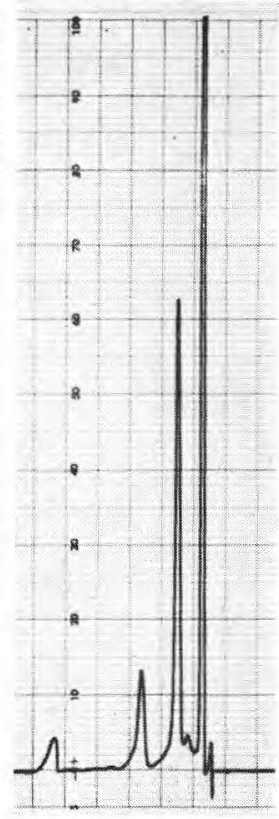

2

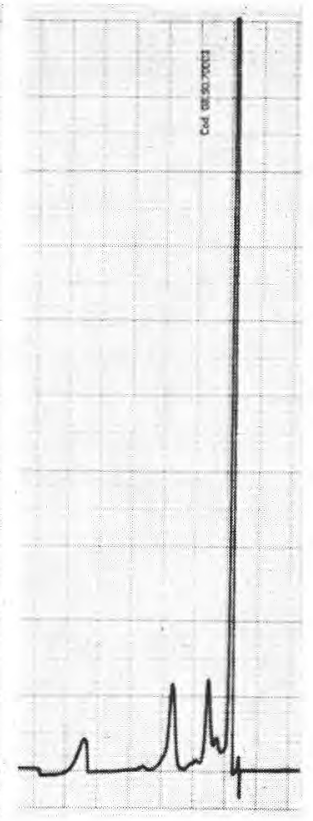

\section{1}

fig. 2

Production d'aldéhyde acétique en lait UHT et lait UHT autoclavé par la souche PRA 10 (L. bulgaricus)

1 = test-lait UHT autoclavé

$2=$ PRA 10 en lait UHT

$3=$ PRA 10 en lait UHT autoclavé

(sensibilité $2 \mathrm{x}$ ) 
TABLEAU 2. - Production d'aldéhyde acétique par chaque souche dans le lait UHT et dans le lait UHT autoclavé

\begin{tabular}{|c|c|c|c|c|c|c|c|c|c|c|}
\hline & \multicolumn{10}{|c|}{ Souches } \\
\hline & CHL 12 & CHL 21 & SH 37 & PRA 10 & PRA 55 & MOR 72 & MOR 77 & STR 45 & STR 63 & ZOL 29 \\
\hline \multicolumn{11}{|l|}{$\mathrm{pH}$ après $24 \mathrm{~h}$ à $42^{\circ} \mathrm{C}$ en : } \\
\hline $\begin{array}{l}\text { a) lait UHT } \\
\text { b) lait UHT autoclavé }\end{array}$ & $\begin{array}{l}4,22 \\
3,70\end{array}$ & $\begin{array}{l}4,25 \\
3,72\end{array}$ & $\begin{array}{l}4,42 \\
3,80\end{array}$ & $\begin{array}{l}4,38 \\
3,75\end{array}$ & $\begin{array}{l}4,38 \\
3,85\end{array}$ & $\begin{array}{l}4,15 \\
3,87\end{array}$ & $\begin{array}{l}4,22 \\
3,82\end{array}$ & $\begin{array}{l}4,40 \\
4,35\end{array}$ & $\begin{array}{l}4,30 \\
4,28\end{array}$ & $\begin{array}{l}4,30 \\
4,12\end{array}$ \\
\hline \multicolumn{11}{|c|}{$\begin{array}{l}\text { Surface en } \mathrm{mm}^{2} \text { du pic de l'aldéhyde } \\
\text { acétique produit en : }\end{array}$} \\
\hline $\begin{array}{l}\text { a) lait UHT } \\
\text { b) lait UHT autoclavé }\end{array}$ & $\begin{array}{l}2080 \\
2150\end{array}$ & $\begin{array}{l}1760 \\
1810\end{array}$ & $\begin{array}{l}1540 \\
1550\end{array}$ & $\begin{array}{r}472 \\
1200\end{array}$ & $\begin{array}{r}330 \\
1240\end{array}$ & $\begin{array}{l}356 \\
475\end{array}$ & $\begin{array}{l}229 \\
418\end{array}$ & $\begin{array}{l}110 \\
368\end{array}$ & $\begin{array}{l}144 \\
272\end{array}$ & $\begin{array}{l}151 \\
261\end{array}$ \\
\hline Classification & L.b. $\left({ }^{*}\right)$ & L.b. & L.b. & L.b. & L.b. & L.b. & L.b. & S.t $(* *)$ & S.t. & S.t. \\
\hline
\end{tabular}

(*) L.b. = L. bulgaricus

${ }^{* *}$ S.t. $=$ Str. thermophilus 
Il y a aussi une augmentation avec les autres souches $L$. bulgaricus comme il y a aussi une légère amélioration en ce qui concerne les trois souches de Str. thermophilus (STR 45, STR 63 et ZOL 29 du tableau 1). PRA 10.

La figure 2 illustre clairement le comportement de la souche

En même temps, on a noté, comme il est reporté dans le tableau 2 que toutes les souches de L. bulgaricus et non celles de Str. thermophilus présentent dans le lait UHT autoclavé un développement beaucoup plus intense avec, par conséquent, une acidification plus grande de la couche sous-jacente en ce qui concerne le lait UHT.

De l'ensemble des révélations faites, on peut donc observer que dans le domaine de l'espèce $L$. bulgaricus on relève des souches qui manifestent dans le lait UHT une capacité diverse pour la production de l'aldéhyde acétique.

A côté des souches faiblement productrices, et elles forment la majorité de celles que nous avons isolées, il en existe d'autres fortement productrices, mais différentes des premières, qui peuvent montrer des capacités similaires aux secondes quand on les fait se développer dans le lait UHT re-stérilisé en autoclave.

Bottazzi, Battistotti et Vescovo [17] avaient déjà mis en évidence que l'intensité du traitement thermique subi par le lait conditionnait, dans les cultures continues, la stabilité du rapport "bacilles-coques » mais on signale maintenant qu'une telle influence s'étend aussi à la capacité de production de l'aldéhyde acétique de la part des souches séparées.

Le fait que, dans le lait UHT, les éléments de $L$. bulgaricus montrent une capacité diverse à produire l'aldéhyde acétique, peut représenter un moyen utile chaque fois que l'on désire choisir les souches à utiliser dans une combinaison pour yaourt.

Il va de soi que celles qui sont en état de produire une quantité élevée d'aldéhyde acétique dans le lait UHT sont certainement d'un plus grand intérêt pratique par rapport aux autres.

\section{B) Accumulation de l'aldéhyde acétique en cultures associées formées de souches faiblement productrices}

Avec la série présente d'essais on a voulu voir le comportement des cultures associées formées de bacilles seuls ou bien de bacilles et de coques des souches faiblement productrices dans le lait UHT, en ayant toujours pour référence la production d'aldéhyde acétique.

Comme première combinaison on a choisi les souches MOR 72 et MOR 77 et en parallèle, les souches PRA 10 et PRA 55, toutes de l'espèce $L$. bulgaricus, enfin comme troisième combinaison les souches STR 45 et ZOL 27 ces dernières appartenant à l'espèce Str. thermophilus. 
Ces trois combinaisons n'ont montré aucune amélioration dans la production de l'aldéhyde acétique en maintenant toujours de basses valeurs.

Quand en revanche on ajoute à ces combinaisons données une souche de Str. thermophilus on a systématiquement une plus grande activité de la forme bacillaire, relevant aussi la valeur du $\mathrm{pH}$ final et une production nettement supérieure d'aldéhyde acétique.

Ce résultat est nettement obtenu aussi avec une combinaison plus simple, soit une souche de L. bulgaricus peu productrice dans le lait UHT et une souche de Str. thermophilus.

Dans le tableau 3 on a voulu réunir les résultats tirés d'une série obtenue avec du lait UHT à la température de $42^{\circ} \mathrm{C}$.

\section{TABLEAU 3}

Production d'aldéhyde acétique en cultures associées dans le lait UHT

\begin{tabular}{|c|c|c|}
\hline $\begin{array}{l}\text { Indication de la } \\
\text { combination }\end{array}$ & $\begin{array}{l}\mathrm{pH} \text { dans le lait UHT } \\
\text { après } 24 \mathrm{~h} \text { à } 42^{\circ} \mathrm{C}\end{array}$ & $\begin{array}{l}\text { Surface en } \mathrm{mm}^{2} \text { du pic } \\
\text { de l'aldéhyde acétique }\end{array}$ \\
\hline $\begin{array}{l}\text { MOR } 72+\text { MOR } 77 \\
\text { MOR } 72+\text { MOR } 77+\text { STR } 45\left(^{*}\right) \\
\text { MOR } 72+\text { STR } 45 \\
\text { PRA } 10+\text { PRA } 55 \\
\text { PRA } 10+\text { PRA } 55+\text { STR } 45 \\
\text { STR } 45+\text { ZOL } 29\end{array}$ & $\begin{array}{l}4,12 \\
3,80 \\
3,85 \\
4,15 \\
3,75 \\
4,20\end{array}$ & $\begin{array}{l}472 \\
832 \\
781 \\
415 \\
874 \\
165\end{array}$ \\
\hline
\end{tabular}

(*) Les souches avec l'indication STR et ZOL appartiennent à l'espèce Str. thermophilus, les autres à l'espèce $L$. bulgaricus.

Les résultats notés sont hautement significatifs et suffisants pour attirer l'attention sur l'importance qu'assument les souches de Str. thermophilus dans la formation de l'association à employer pour la production du yaourt.

Les formes coques non seulement contribuent, comme l'a rappelé récemment Martens [18], à caractériser le caillot au point de vue physique, à permettre un développement plus grand et plus rapide des formes bacillaires et cela afin d'être en mesure d'exercer une hydrolyse partielle des protéines du lait et de libérer de petites quantités d'acide formique, comme l'ont démontré Veringa, Galesloot et Davelar [19], mais encore concourent certainement à créer une ambiance favorable afin que, dans le lait UHT, ces souches de L. bulgaricus faiblement productrices d'aldéhyde acétique deviennent bonnes productrices. Cela confirme les vastes relations symbiotiques 


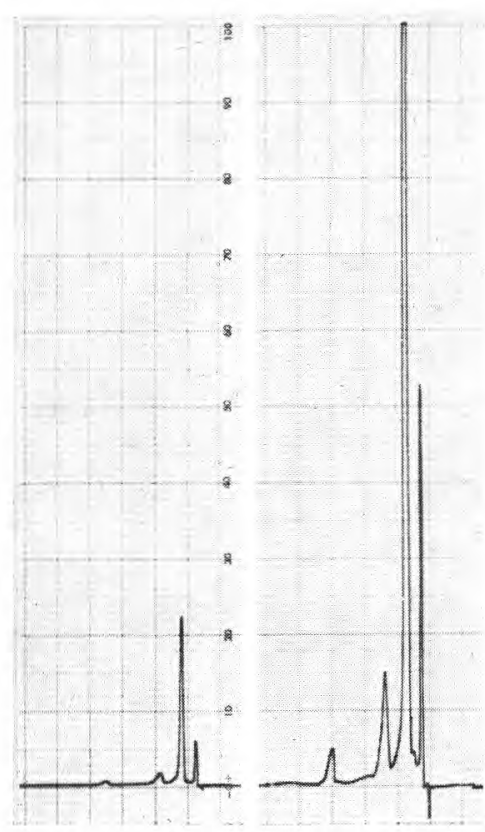

$5 a$

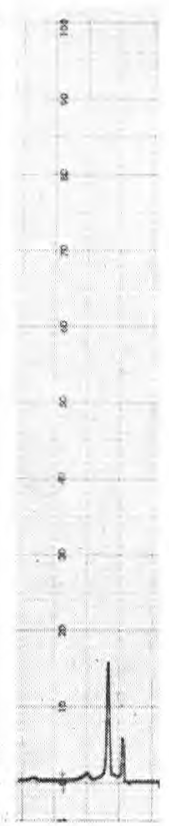

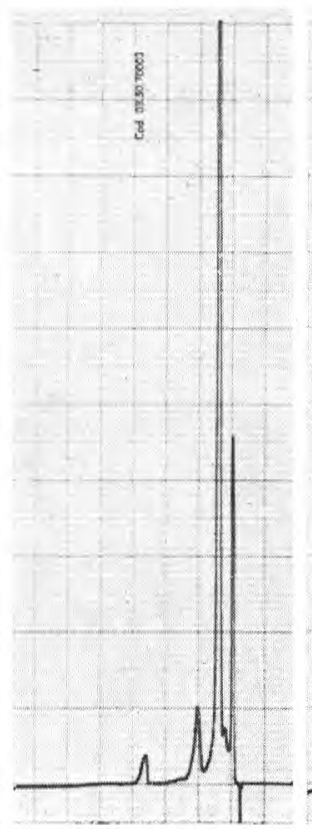

4

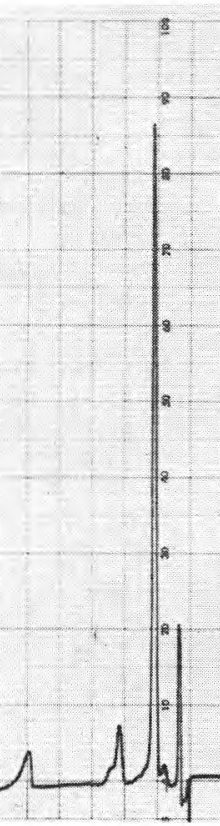

3

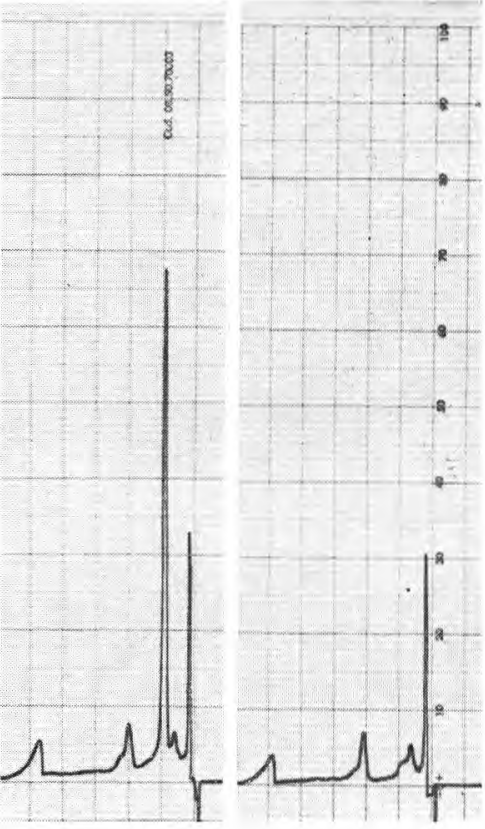

fig $3 A$

Marche de la production de l'aldéhyde acétique pendant la fabrication du yaourt

$$
\begin{aligned}
& 1=\text { test-lait UHT traité à } 90^{\circ} \mathrm{C} \times 30 \mathrm{mn} ; \\
& 2=\text { yaourt après } 2 \mathrm{~h} \text { et } 30 \mathrm{mn}(\mathrm{pH} \mathrm{4,85);} \\
& 3=\text { yaourt après } 3 \mathrm{~h} \quad(\mathrm{pH} \mathrm{4,50)} ; \\
& 4=\text { yaourt après } 3 \mathrm{~h} \text { et } 30 \mathrm{mn}(\mathrm{pH} \mathrm{4,35);} \\
& 5=\text { yaourt après } 4 \mathrm{~h} \\
& (1,2,3,4 \text { et } 5 \text { sensibilité } 2 \mathrm{x}) ;
\end{aligned}
$$

( $4 \mathrm{a}$ et $5 \mathrm{a}$ sensibilité $16 \mathrm{x}$ ). 


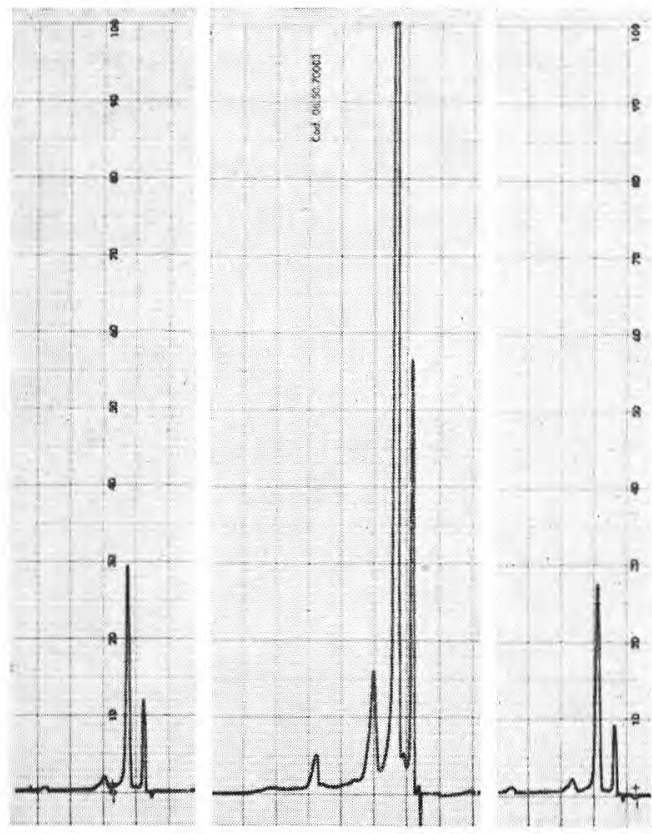

$9 a$
9
$8 \mathrm{a}$

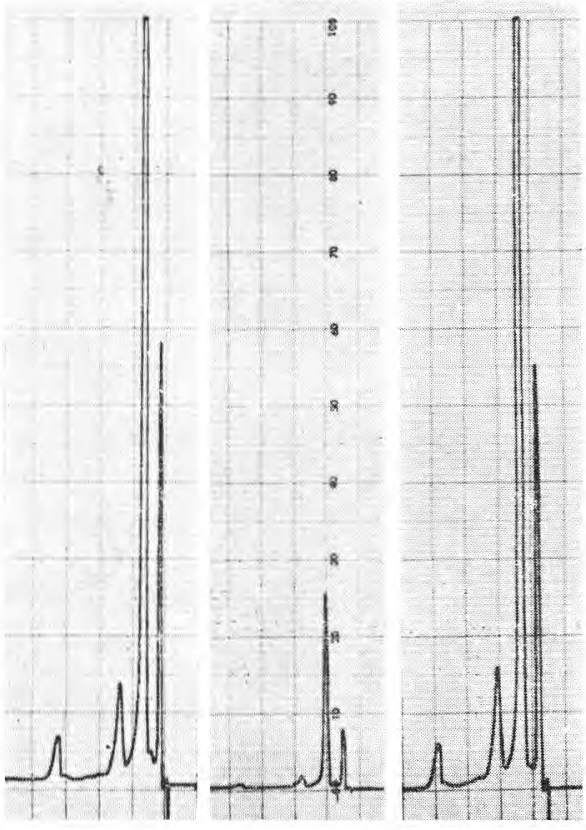

8

$7 a$

\begin{abstract}
7
\end{abstract}
.

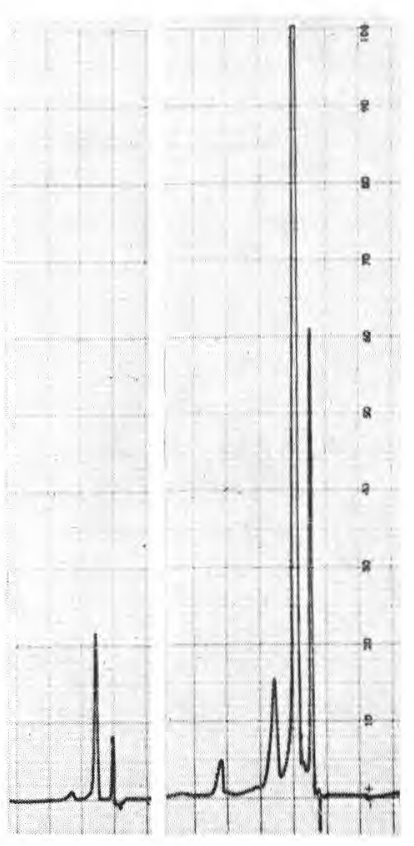

$6 a$

6

fig. $3 B$

Marche de la production de l'aldéhyde acétique pendant la fabrication du yaourt

$$
\begin{array}{ll}
6=\text { yaourt après } 4 \mathrm{~h} \text { et } 30 \mathrm{mn} & (\mathrm{pH} 4,20) \\
7=\text { yaourt après } 5 \mathrm{~h} & (\mathrm{pH} 4,18) ; \\
8=\text { yaourt après } 6 \mathrm{~h} & (\mathrm{pH} 4,10) ; \\
9=\text { yaourt après } 7 \mathrm{~h} & (\mathrm{pH} 4,05) ; \\
(6,7,8 \text { et } 9 \text { sensibilité } 2 \mathrm{x}) ; &
\end{array}
$$

(6a, 7a, 8a et $9 \mathrm{a}$ sensibilité $16 \mathrm{x})$. 
existant entre les deux organismes, comme déjà rappelé par Pette et Lolkema [20], Bautista, Dahiya et Speck [21] et, plus récemment, Hamdan, Kunsman et Deane [22].

Les résultats que l'on peut atteindre, d'un point de vue pratique dans la production du yaourt sont certainement liés à une large connaissance des caractéristiques des souches séparées composant l'association, non seulement dans le rapport entre souches de bacilles et de streptocoques, les conditions d'inoculation et d'incubation et de conduite de la fermentation mais encore, à notre avis, dans les résultats expérimentaux ci-dessus présentés.

\section{C) Production d'aldéhyde acétique pendant la préparation du yaourt}

La marche de l'accumulation de l'aldéhyde acétique pendant la fabrication du yaourt a été suivie dans le lait UHT re-traité pendant $30 \mathrm{mn}$ à $90^{\circ} \mathrm{C}$ et inoculé par une combinaison de plusieurs souches des espèces $L$. bulgaricus et Str. thermophilus que l'on a voulu préparer sur la base des connaissances précédentes.

Les contrôles ont été commencés après 2 h 30 d'incubation à $42^{\circ} \mathrm{C}$ et prolongés jusqu'à la $7^{\mathrm{e}}$ heure. Les résultats obtenus sont rassemblés dans les figures $3 \mathrm{~A}$ et $3 \mathrm{~B}$.

On peut dire que la production de l'aldéhyde acétique commence à être évidente quand le $\mathrm{pH}$ du yaourt atteint la valeur de 4,90 c'està-dire après environ 2 h 30 d'incubation, pour augmenter ensuite plus rapidement jusqu'à ce qu'il atteigne la valeur de 4,05 après $7 \mathrm{~h}$, mais la quantité d'aldéhyde acétique ne présente plus, dans les dernières heures, d'augmentation significative.

Comme on peut le voir des chromatogrammes rapportés, l'acétone et l'alcool, qui sont déjà présents en petites quantités dans le lait de départ, ne subissent pas au contraire de variations notables pendant la fabrication du yaourt.

\section{Résum é}

Dans l'appréciation des qualités du yaourt, la saveur acquiert une importance au même titre que la consistance et la crémosité.

Dans les limites de ce cadre, on a effectué des recherches tendant à connaître l'influence qu'exercent, sur la production de l'aldéhyde acétique, les souches seules et associées composant la culture pour yaourt en liaison avec les traitements thermiques du lait.

L'enquête s'est développée avec un certain nombre de souches isolées du yaourt du commerce, sur lesquelles furent d'abord faits, pour leur référence taxonomique, des essais physiologiques, sérologiques et de composition du DNA puis, en deuxième lieu, en utilisant la technique "Head Space " gaz chromatographie, on a évalué la capacité de production de l'aldéhyde acétique. 
Des résultats obtenus on a pu souligner l'importance que revêt pour la production du goût, le choix de la souche aromatique, sa combinaison avec les streptocoques thermophiles et la nature des traitements thermiques du lait.

De nombreuses souches de L. bulgaricus dans le lait UHT se sont révélées faiblement productrices d'aldéhyde acétique tandis que les mêmes en produisent des quantités supérieures quand elles sont ensemencées dans le lait UHT re-stérilisé en autoclave ou quand elles sont associées avec des souches de Str. thermophilus.

De l'ensemble des indications obtenues en ce qui concerne les caractéristiques des souches singulières on a procédé enfin à la préparation d'une culture associée pour yaourt composée de plusieurs souches des deux espèces et on a vu, ceci aboutissant certainement à une signification pratique, que la production d'aldéhyde acétique ne commence pas avant d'avoir atteint la valeur de $\mathrm{pH}$ 5,00 et augmente rapidement tant que le $\mathrm{pH}$ du lait en fermentation n'atteint pas $4,30,4,20$.

Après cette valeur atteinte en 3 h 30 environ d'incubation à $42^{\circ} \mathrm{C}$, la quantité d'aldéhyde acétique augmente encore légèrement pour se stabiliser ensuite quand le $\mathrm{pH}$ est proche de 4,00.

\section{S u m m a r y}

In evaluating yoghurt quality, besides its consistence and cream appearance, great importance is attributed also to the " yoghurt flavour ».

In this context we have carried out some researches volved to investigate about the influence exerted by single and associated strains of yoghurt culture, in combination with milk heat treatments, on acetic aldehyde production.

The research has been undertaken on several strains isolated from commercial yoghurt : at the first, in order to have a taxonomic reference, physiological, serological tests and DNA base composition have been made, and after, applying the gas chromatographic head space technique, the ability to produce acetic aldehyde has been evaluated.

From the obtained results it has been shown the importance, for flavour production, of aromatizing strain kind, its association with thermophilic streptococci and milk different heat treatments.

Many strains of $L$. bulgaricus in UHT sterilized milk are week in acetic aldehyde production, while they produce a great quantity of it when grown in UHT autoclave resterilized milk, or when they are associated to some strains of Str. thermophilus.

From the knowledges obtained as regards single strains characteristics, we have prepared a yoghurt culture with associated strains ; 
from the results, also having a pratical significance, acetic aldehyde productions begins at $\mathrm{pH} 5,00$ and increases quickly until $\mathrm{pH}$ reaches $4,40-4,30$ values.

After this value, reached in about three hours at $42^{\circ} \mathrm{C}$, acetic aldehyde amount increases little, and stabilizes when $\mathrm{pH}$ is about 4,00 value.

Reçu pour publication en décembre 1972

de l'Istituto di Microbiologica Lattiero-casearia de Piacenza.

\section{Remerciements}

Les auteurs remercient le docteur Dellaglio et le docteur Sarra pour la précieuse collaboration qu'ils leur ont donnée pendant l'exécution des essais taxonomiques concernant les micro-organismes employés.

\section{Bibliographie}

[1] Grigoroff (S.) (1905). - Rev. Méd. Suisse Romande, 25, 714.

[2] Kuntze (W.) (1908). - Zbl. Bact., II, 21, 737-768.

[3] Orla Jensen (S.) (1919). - Mem. Acad. Roy. Sci. Let., Copenhague Sci., 8, $\mathrm{V}, 79$.

[4] Pette (J. W.) et Lolkema (H.) (1950). - Neth. Milk Dairy J., 4, 261.

[5] Bottazzi (V.) et Dellaglio (F.) (1967). - J. Dairy Res., 34, 109.

[6] Schulz (M. E.) et Hingst (G.) (1954). - Milchw., 9, 330.

[7] Schulz (M. E.), Voss (E.) et Kley (W.) (1954). - Milchw., 9, 361.

[8] Turcic (M.), Rasic (J.) et Canic (V.) (1968). - Hemijska Ind. (Tehnika), $22,872$.

[9] Gorner (F.), Palo (V.) et Bertan (M.) (1968). - Milchw., 23, 94.

[10] De Man (J. C.), Rogosa (M.) et Sharpe (M. E.) (1960). - J. Appl. Dact., 23, 130.

[11] Politi (I.) (1965). - Ann. Microbiol., 15, 31.

[12] Marmur (J.) (1961). - J. Mol. Biol., 3, 208.

[13] De Ley (J.) (1970). - J. Bact., 101, 738.

[14] Sharpe (M. E.) (1955). - J. Gen. Microbiol., 12, 107.

[15] Jennings (W. G.), Viljhalmsson (S.) et Dunkley (W. L.) (1962). - J. Food Sci., 27, 306.

[16] Bassette (R.), Ozeris (S.) et Whitnah (C. H.) (1962). - J. Food Sci., $28,84$.

[17] Bottazzi (V.), Battistotti (B.) et Vescovo (M.) (1971). - Michw., 26, 214.

[18] Martens (R.) (1972). - Rev. de l'Agricol., 25, 461.

[19] Veringa (H. A.), Galesloot (T. E.) et Davelar (H.) (1968). - Neth. Milk Dairy $J ., 22,114$.

[20] Pette (J. W.) et Lolkema (H.) (1950). - Neth. Milk Dairy J., 4, 209.

[21] Bautista (E.S.), Dahiya (R.S.) et Speck (M. L.) (1966). - J. Dairy Res., 33, 299.

[22] Hamdan (J. Y.), Kunsman (J. E.) et Deane (D. D.) (1971). - J. Dairy Sci., $54,1080$. 